\title{
Effect of anthropogenic light on bird movement, habitat selection, and distribution: a systematic map protocol
}

\author{
Carrie Ann Adams ${ }^{1 *}$, Arden Blumenthal ${ }^{2}$, Esteban Fernández-Juricic ${ }^{2}$, Erin Bayne ${ }^{1}$ and Colleen Cassady St. Clair ${ }^{1}$
}

\begin{abstract}
Background: Anthropogenic light is known or suspected to exert profound effects on many taxa, including birds. Documentation of bird aggregation around artificial light at night, as well as observations of bird reactions to strobe lights and lasers, suggests that light may both attract and repel birds, although this assumption has yet to be tested. These effects may cause immediate changes to bird movement, habitat selection and settlement, and ultimately alter bird distribution at large spatial scales. Global increases in the extent of anthropogenic light contribute to interest by wild life managers and the public in managing light to reduce harm to birds, but there are no evidence syntheses of the multiple ways light affects birds to guide this effort. Existing reviews usually emphasize either bird aggregation or deterrence and do so for a specific context, such as aggregation at communication towers and deterrence from airports. We outline a protocol for a systematic map that collects and organizes evidence from the many contexts in which anthropogenic light is reported to affect bird movement, habitat selection, or distribution. Our map will provide an objective synthesis of the evidence that identifies subtopics that may support systematic review and knowledge gaps that could direct future research questions. These products will substantially advance an understanding of both patterns and processes associated with the responses of birds to anthropogenic light.

Methods: The protocol describes the steps taken to ensure the search for evidence is comprehensive, transparent and replicable. We will find relevant studies in the grey and peer-reviewed literature using publication databases, Google Scholar, stakeholder suggestions, and organizational websites. We will select studies for inclusion in the map by identification of relevant: (i) population including any species of bird; (ii) intervention or exposure to anthropogenic light; and (iii) outcomes including changes in bird movement, habitat occupancy, population density, or distribution. We will extract and organize metadata into a systematic map that can support subsequent search by interested individuals. The quantity of evidence on particular topics will be characterized through heat maps and narrative syntheses, but subsequent work will be needed to evaluate evidence validity.
\end{abstract}

Keywords: Light pollution, Artificial light, Wildlife conservation, Evidence synthesis, Human-wildlife conflict, Behaviour, Wildlife deterrents, Bird strike, Nocturnal migration, Avian mortality

\footnotetext{
*Correspondence: caadams1@ualberta.ca

${ }^{1}$ Department of Biological Science, University of Alberta, CW 405,

Biological Sciences Building, Edmonton, AB T6G 2E9, Canada

Full list of author information is available at the end of the article
} 


\section{Background}

Artificial light has been increasing globally at a rate of $2.2 \%$ per year [1], with a high diversity of documented effects on wildlife, including birds. Man-made objects (buildings, vehicles, power lines, street lighting, etc.) have spectral properties (e.g. wavelength, intensity) that differ from natural light present in avian evolutionary history. In many contexts, birds aggregate near artificial lights at night, with documented cases dating back to the nineteenth century (reviewed by [2]) that are assumed to stem from attraction to light. Paradoxically, light is also sometimes used to deter birds from zones of humanwildlife conflict (reviewed by [3]). There is evidence that both the potential attractant and deterrent effects of light can cause immediate changes to bird movement [4] and alter habitat selection and settlement at a local $[5,6]$ and regional spatial scale [7]. The potential of light as a management tool has generated interest in a wide variety of disciplines, from pest management to bird conservation $[6,8]$. Despite being applicable in many contexts, there has been no attempt to unite the evidence showing attraction, deterrence, and neutral responses of birds to light. Integrating knowledge related to how birds respond to anthropogenic light could contribute substantially to our basic understanding of relevant physiological and behavioural mechanisms. In turn, this understanding has tremendous potential to minimize bird mortalities and sublethal effects resulting from illuminated structures and regional light pollution, and also maximize bird deterrence in zones of human-wildlife conflict.

Despite high diversity of light effects on birds and large variation in their temporal and spatial scales, there has been no broad synthesis of this literature. Existing peerreviewed syntheses have tended to emphasize specific effects of light, such as disruption to migration [9]. Similarly, existing reviews in the grey literature target particular contexts for bird control, such as agriculture [3], poultry [10], mining [11] and aviation [12]. Such specific contexts are also targeted by some reviews in the peerreviewed literature, such as offshore oil and gas [13], wind turbines [14], and communication towers [15]. The most comprehensive review of night lighting to date [2] synthesizes evidence from the grey and peer-review literature, but it is now over 10 years old and did not consider the deterrent effects of light. There is both opportunity and need for a comprehensive synthesis of the effects of anthropogenic light on birds.

Such a synthesis is necessary to develop new insights into how to mitigate the disruptive effects of light on birds, which most often applies to steady-burning lights (i.e. lights with constant luminous intensity) and nocturnally-migrating birds. Anthropogenic light has been associated with all major sources of collision mortality
[13, 15-18]: buildings and windows [17], transmission lines [15], roads [18], and communication towers [15]. Light may increase bird numbers and non-linear flight paths at illuminated structures $[13,15,17,18]$ and affect both movement and distribution at large spatial scales $[7,19]$. Even sub-lethal effects of aggregation and disruption to flight paths may ultimately reduce survival during migration by reducing energy stores and delaying arrival at breeding or wintering grounds [2]. Despite documented effects of light, some studies show no significant effect of light on bird flight behavior, density, or mortality at illuminated man-made structures [20, 21]. Describing the numbers of studies and contexts associated with different kinds of light effects is a necessary first step toward potential mitigation.

Reducing the disruptive effects of artificial light requires an understanding of the contexts, light characteristics, and species involved in which bird aggregation is most likely to occur. A map of the types and correlates of light effects on birds will advance subsequent studies of mechanisms. For example, a subsequent review may reveal that aggregation is more likely for some bird species, spectral characteristics of lights source, weather, and ambient light conditions. Such factors may explain why one study reported that red lights are less likely to cause aggregation of nocturnal migrants [22], while another study found that green and blue lights were less disruptive to birds than red ones [23]. Similarly, ambient light may explain why migrating eiders (Somateria mollissima and S. spectabilis) exposed to artificial light increased flight velocity during the day, but decreased velocity at night [20]. Bird aggregation and mortality is often documented during nights of low cloud cover [23, 24], but sometimes occurs in clear weather as well [19]. Comparison of all available evidence and further primary research is needed to understand the many factors that contribute to bird aggregation around artificial light sources. Current context-dependent reviews of light effects are likely to overlook promising explanatory factors that may be revealed from broader syntheses.

The deterring effects of light are similarly scattered in the existing literature. Many industrial contexts now employ flashing lights, rotating beams, and lasers to deter birds from hazards, but there has been no synthesis of their efficacy. Such a synthesis would be relevant to applications that include agricultural lands, aquaculture facilities, airports, urban structures, industrial ponds, and other contexts in which birds cause damage, pose a danger to humans, or may be harmed by associated anthropogenic activity (reviewed by [3, 6]). Light deterrents potentially offer a significant advantage over chemical and acoustic deterrents because they (a) are non-lethal, (b) can target a specific area, (c) can be used 
around other man-made structures, and (d) are silent, avoiding disruption to wildlife and neighboring humans associated with noise pollution [25]. However, the way these studies have measured avoidance has been recently questioned, emphasizing the need for choice tests to establish whether birds are indeed avoiding lights in the true behavioral sense [26].

As for the literature associated with bird aggregation and attraction to light, there are few generalizations with which to predict or evaluate the effect of light as bird deterrents. For example, a particular light-based treatment may effectively deter some bird species and not others $[6,25]$, a deterrent that was initially effective may no longer induce a reaction after birds habituate [25], and small scale movements by birds as pests may simply relocate them to nearby areas $[27,28]$. The literature reflects a general consensus that some types of lasers and strobes can deter some bird species in some contexts [3, $10,12]$, but no systematic comparison has been applied to understand when and why particular treatments are effective. There is similarly no synthetic evaluation of the risk to the eyes of people and wildlife posed by using lasers, which can cause temporary or permanent damage to vision [30-32].

Incorporating laboratory and controlled field studies in our Systematic Map will provide evidence for understanding the physiological, ecological and evolutionary mechanisms governing bird responses observed in field studies. Understanding these mechanisms will in turn support better design of lighting and illuminated structures. The physiological and cognitive basis for avian responses to light remains elusive $[2,8,29]$, but laboratory studies offer some insight into how anthropogenic light may disorient birds. Early laboratory studies suggested that bright lights in dark environments often disorient birds by decreasing their ability to see the environment surrounding the light source (reviewed by [29]), and lights of particular wavelengths or intensities disrupt their ability to sense the earth's magnetic field [33, 34]. Disorientation in response to loss of night vision or magnetoreception has been referenced by authors of field studies as an explanation for aggregation around anthropogenic light $[19,23,29]$, but the mechanism by which disoriented birds are attracted to fly towards and remain within the illuminated area is unclear. The systematic map will integrate relevant evidence from field and lab studies necessary to support inferences about how potential mechanisms of disorientation demonstrated in controlled settings may influence bird behaviour in field studies where aggregation is observed.

The mechanisms associated with the deterring effects of light on birds are also obscure and could benefit from better integration of lab and field studies. Predicting bird behavioural response to light-based deterrents requires both an understanding of what the bird is seeing, based on the target species' visual system, and how the bird is likely to respond once the stimuli is perceived [35]. Bird perception is difficult to predict because the avian visual system differs in several ways from that of humans (summarized in [36-39]) and visual perception varies across bird species [35]. Given that a bird perceives the light deterrent, it may respond with movement to prevent collision with an approaching object (e.g. airplane or vehicle) or avoid a novel stimuli (e.g. moving laser) [40]. This systematic map will include empirical evidence from laboratory and field studies documenting bird reaction to deterrents across a range of species and deterrent type. The resulting evidence base will support review questions about deterrent efficacy for particular target species or populations, light characteristics, contexts, and intended behavioural responses.

There is a tremendous need for systematic review of many aspects of light effects on birds to inform evidence-based lighting policy and wildlife management. As an example of the link between evidence and policy, research on the disruptive effects of steady-burning light on nocturnally-migrating birds caused changes to regulations set by the US Federal Aviation Administration for obstruction lighting, eliminating non-flashing lights. Substantial voluntary changes in light use were encouraged by Audubon's Light Out programs at cities across North America [41, 42]. Our systematic map will deepen the evidence base for these programs and encourage development of new ones by documenting effective interventions. Others have shown how regulation is limited by inadequate information. For example, the US Bureau of Ocean Energy Management evaluation of lighting schemes identified inconsistency in research addressing the reaction of birds to light of varying wavelengths (i.e. color), demonstrating a need for systematic review of the evidence [43]. Bird management at airports, tailings ponds, urban areas, and agricultural fields would benefit from systematic review of light-based deterrents.

The secondary questions of our systematic map include three main subtopics of interest to bird management and conservation: (a) bird aggregation around artificial light sources, (b) bird deterrence by light-based interventions, and (c) avian habitat use and distribution in artificially illuminated landscapes. These three subtopics are linked by overlapping evidence bases, with many studies applying to more than one subtopic, and mechanistic explanations. For example, a study may document both aggregation and dispersal effects if comparing flashing and steady-burning light treatments. The evidence base for dispersal and habitat selection overlaps where the efficacy of light-based deterrents affects bird habitat use in 
landscapes where deterrents are employed [44]. Similarly, changes in flight path induced by bright light sources may affect migratory stopover habitat use [7]. In all cases, bird response is likely to depend on light characteristics as perceived by the bird and the ambient light conditions, life history stage and taxa of the bird, and the spatial and temporal scale of the intervention. The systematic map will provide a comprehensive database of all the available evidence, with metadata on the sources of heterogeneity across studies, with the dual goals of (a) providing a comprehensive list of light-based interventions and evidence of their efficacy in various contexts and (b) identifying subtopics that may support a systematic review.

\section{Stakeholder engagement}

Informal conversations with stakeholders from industries, government agencies, and non-profit organizations confirmed that this topic is relevant in many fields. We developed a questionnaire for stakeholders to expand our literature search, identify secondary questions of particular importance, support map interpretation, and guide subsequent systematic reviews and primary research (Additional file 1). To date, we have received responses from four stakeholders. We will continue to target individuals with expertise in the fields of bird conservation and management, including human-avian conflict, aiming for a total of 12 responses. Stakeholders include leaders and researchers at universities or nonprofit organizations associated with bird conservation or management, in addition to wildlife managers at federal, provincial, and civic authorities. We will search grey literature sources suggested by stakeholders for relevant articles and listed in the published map. We will also provide the opportunity for stakeholders to review the final map and request that they share the resulting database with relevant decision makers.

\section{Objective of the review}

The objective of this systematic map is to provide an overview of the evidence of the effects of anthropogenic light on bird movement, distribution and habitat selection. The map will integrate light effects associated with attraction and aggregation as well as deterrence and dispersal of birds. We will include studies documenting the effects of multiple light sources and identify covariates of effects that may contribute to variation in bird responses to light. Possible covariates include light characteristics (e.g. wavelength, intensity, direction, and flashing pattern), environmental variables (e.g. weather variables, temporal variables, moon phase, land/freshwater/ocean), and population characteristics (e.g. species, bird activity during intervention, domestication status, migratory status). The map will describe the quantity of evidence available on each subtopic, without evaluating the validity of the evidence presented. The map will identify areas of primary concern for managers, topics for further primary research, and potential subtopics for systematic reviews.

\section{Primary question}

What is the evidence that anthropogenic light affects bird movement, habitat selection, and distribution?

\section{Secondary questions}

- What information is available documenting that artificial illumination is associated with bird aggregation or attraction?

- What information describes the effect of light as a means of deterring or dispersing birds?

- What are the contexts in which anthropogenic light is associated with changes (either increase or decrease) in bird habitat use and/or density?

- What documentation is available concerning how the effects of light on birds change over time or space, which may occur as a function of prior exposure, weather conditions, light characteristics, or other factors?

Components of the primary question

\begin{tabular}{ll}
\hline Population (P) & All bird species \\
Intervention or exposure (I or E) & Anthropogenic light sources \\
Comparator (C) & $\begin{array}{c}\text { Similar habitats, structures, or } \\
\text { landscapes that are unlit or lit with } \\
\text { different types of light; same study } \\
\text { site before or after intervention } \\
\text { Changes in bird movement, habitat } \\
\text { occupancy, population density, or } \\
\text { distribution }\end{array}$
\end{tabular}

See Article Screening and Eligibility Criteria for more detailed descriptions of each component.

\section{Methods}

This systematic map follows CEE guidelines [45] and ROSES reporting standards [46].

\section{Searching for articles}

\section{Database and citation indexing service search strategy}

The databases outlined in Table 1 will be searched for articles relevant to our primary question. We chose Web of Science Core Collection as our primary tool for its comprehensive coverage of the published literature. We determined that the maximum number of articles we can 


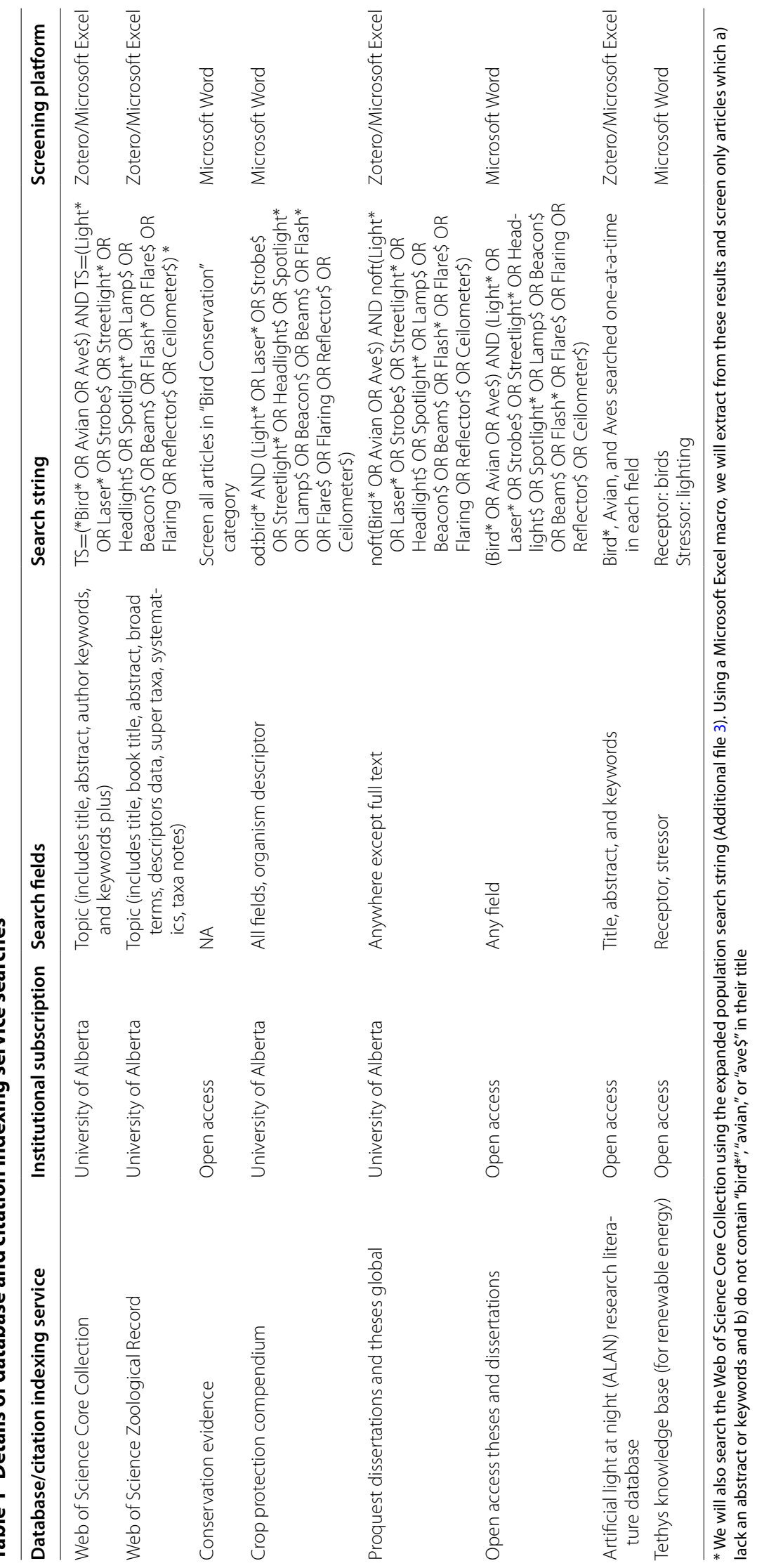


screen is 20,000, which made it feasible to add only one other comprehensive citation indexing service. We chose Web of Science Zoological Record because it indexed six of the fourteen benchmark articles that were not indexed in the Web of Science Core Collection (Additional file 2). We found all six of these articles using the proposed search string in the Web of Science Zoological Record (Table 2). Only three of the benchmark articles not indexed in Web of Science Core Collection were available in Scopus and none were available in JSTOR. Additionally, we will search Proquest Dissertations and Theses and Open Access Theses and Dissertations to find relevant graduate theses that may not be indexed in Web of Science.

\section{Search string}

The mapping team developed a list of search terms for the population and intervention components of the primary question based on terms used in a list of "benchmark articles" (Additional file 2). We describe the search strings for Web of Science Core Collection below. Modifications to this search string for other databases and citation indexing services are listed in Table 1.

Intervention/exposure The search terms for the relevant interventions/exposures were consistent across the test articles. In additional to the terms outlined in Table 2, the terms "reflect" and "LED" were also considered for inclusion in the intervention/exposure search string, but removed because of they were commonly used in unrelated contexts. Our search strategy will find all benchmark articles that used these terms to describe an intervention because these studies also included the word "light" in their titles or abstracts. We considered including an additional intervention/exposure string including terms similar to "anthropogenic", but found that some benchmark studies did not include any such terms in their titles, keywords, or abstracts.

Population During scoping, we developed the population string "*bird" OR avian OR ave\$" to search the Web of Science Core Collection (Table 2). Because we were concerned that some relevant articles in the Web of Science Core Collection may not be found using this popula- tion string, hereafter called the "proposed search string," we tested a search string that included all of the common family names listed by the International Ornithological Conference World Bird List 2018 [47], hereafter called the "expanded search string" (Additional file 3). Using the expanded population string and the intervention/exposure string described above, Web of Science Core Collection returned 35,767 results. CAA screened the first 4000 articles from this search (sorted by date) and an additional 2000 papers selected randomly from all search results, identifying 37 eligible articles. These eligible articles were added to the benchmark articles list. The benchmark articles list contains 64 known eligible articles that are contained in the Web of Science Core Collection, hereafter called the "known eligible articles." We searched the Web of Science Core Collection using the proposed search string ("bird" OR avian OR ave\$), generating 10,846 results. We searched within these results for each of the 64 known eligible articles and found 59 of these articles with the proposed population search string.

All five of the eligible articles that were not found by the proposed search string lacked an abstract in the Web of Science Core Collection. When using the proposed population search string instead of the expanded population search string, we may fail to find eligible articles that do not contain "bird," "avian," or "aves" in their title and do not have an abstract in the Web of Science Core Collection. To ensure that we find such articles, we will download all of the search results returned using the expanded population string as a text file, import them into Microsoft Excel, and use a macro to extract all articles that (a) lack abstracts or lack keywords and (b) do not contain "*bird", "avian," or "ave\$" their titles. These articles will be added to the other Web of Science Core Collection search results for eligibility screening. Every step of this process will be carefully documented and published as a supplement to the Systematic Map, including Microsoft Excel macros.

We will search the Web of Science Zoological Record using only the proposed search string (Table 2). All benchmark articles indexed in the Zoological Record were found using the proposed search string. Even articles that lacked an abstract or keywords and did not contain "bird"," "avian," or "ave\$" in their title were found

\section{Table 2 Population and intervention search strings for Web of Science Core Collection and Zoological Record}

\begin{tabular}{ll}
\hline Population & *Bird* OR Avian OR Ave\$ \\
\hline Intervention/exposure & $\begin{array}{l}\text { Light* OR Laser* OR Strobe\$ OR Streetlight* OR Headlight\$ OR Spotlight* OR Lamp\$ OR Beacon\$ OR Beam\$ OR Flash* OR } \\
\text { Flare\$ OR Flaring OR Reflector\$ OR Ceilometer\$ }\end{array}$
\end{tabular}

The "*" is used to represent the addition of any number of characters (inclusive of zero), while the "\$" is used to represent that addition of zero or one character to the search term. We will also search the Web of Science Core Collection using the expanded population search string (Additional file 3). Using a Microsoft Excel macro, we will extract from these results and screen only articles that a) lack abstracts or keywords and b) do not contain "*bird*," "avian," or "ave\$" in their titles 
because the Zoological Record "Topic" field includes Super Taxa, Systematics, and Taxa Notes. The proposed search string found these articles because they contained "Aves" in at least one of the taxa fields.

Outcome During scoping, the mapping team determined that the terms used to describe outcomes in relevant articles were too numerous and unpredictable to include in the search string.

Increasing specificity During scoping, we found that many of the articles in Web of Science Core Collection search results did not address relevant interventions/ exposures, but were found because they contained the phrases, "in light of," "sheds light on," and other iterations of these phrases. We developed a strategy to eliminate from the Web of Science search results any articles that contain an intervention/exposure term within one of these phrases, but do not contain an intervention/exposure term anywhere else in the title, abstract, keywords, or category. We will export our results from Web of Science as comma-delimited text files and open them in Microsoft Excel. We will use an Excel macro to automatically find and replace these phrases with synonyms in capital letters that do not contain an intervention/exposure term (Table 3). This Macro will be published as a supplement to the Systematic Map. After these replacements have been made, we will use a macro to search within the Web of Science search results for articles that contain one of the intervention/exposure search terms in any of the following categories: title, abstract, author keywords, Web of Science keywords, journal name, or Web of Science category. We will exclude from further screening any articles that do not contain an intervention/exposure search term after the irrelevant phrases have been replaced. All search results excluded through this process will be reported in the search records. We will screen ten percent or 200 (whichever is greater) of the excluded articles to ensure that this process did not exclude relevant articles from screening. We will eliminate duplicate articles using Microsoft Excel Remove Duplicates feature by identifying articles with identical authors, titles, and journal names.

\section{Web-based search engines}

We will search Google Scholar for relevant literature using two search strings:

- Bird AND (Light" OR Laser* OR Streetlight* OR Headlight* OR Spotlight\$ OR Lamp\$ OR Beacon\$ OR Beam\$ OR Flash* OR Flare\$ OR Flaring OR Reflector\$)

- Avian AND (Laser" OR Strobe* OR Light" OR Streetlight* OR Headlight* OR Spotlight\$ OR Lamp\$ OR
Table 3 Irrelevant phrases containing search terms in intervention/exposure search string

\begin{tabular}{ll}
\hline Find & Replace \\
\hline In light of & Considering \\
In the light of & Considering \\
Come to light & Reveal \\
Comes to light & Reveals \\
In this light & From this perspective \\
Lightweight & Not heavy \\
Shed light on & Show \\
Shed some light on & Show \\
Sheds light on & Shows \\
Sheds some light on & Shows \\
Light-level geolocator & LL geolocator \\
Light level geolocator & LL geolocator \\
Light-logging geolocator & LL geolocator \\
Light-logger & LL geolocator \\
Light-based geolocator & LL geolocator \\
Light rail & $L$ rail \\
Light-rail & L-rail
\end{tabular}

Irrelevant phrases are listed in the "find" column. In the Web of Science search results, these phrases will be replaced by the text in the "replace" column using a Microsoft Excel macro

\section{Beacon\$ OR Beam\$ OR Flash* OR Flare\$ OR Flaring} OR Reflector\$)

We will search the full text, rather than the title, because several benchmark articles do not include "bird" or "avian" in their titles. We will export and screen the first 1000 results of each Google Scholar search to identify articles that might have been missed with our other search methods.

\section{Organisational websites}

To avoid introducing bias in our map associated with bird type, light contexts, or other factors, we have attempted to create a list of relevant organizations, including NGOs, government agencies, and for-profit companies interested in a diversity of effects of light on birds (Table 4). We will also search additional organisational websites suggested by stakeholders.

\section{Document/file formats}

The search will not have any document type restrictions. If software to open a file is not available, we will request an alternative format from the authors.

\section{Computer settings}

To inhibit the narrowing of searches that could result from learning algorithms built into internet browsers, 
Table 4 Organizational websites included in search

\begin{tabular}{|c|c|c|c|}
\hline Organization & Type & Context & Website \\
\hline Bird Control Group & For-profit & Bird deterrent manufacturer & https://birdcontrolgroup.com/ \\
\hline Bird-X & For-profit & Bird deterrent manufacturer & https://bird-x.com/ \\
\hline Accipiter & For-profit & Bird deterrent manufacturer & https://www.accipiterradar.com/ \\
\hline Bird Avert & For-profit & Bird deterrent manufacturer & http://www.birdavert.com/ \\
\hline DeTect & For-profit & Bird deterrent manufacturer & http://detect-inc.com/ \\
\hline Merlin Environmental & For-profit & Bird deterrent manufacturer & $\begin{array}{l}\text { http://www.merlinenvironmental.co.uk/bird-contr } \\
\text { ol/ }\end{array}$ \\
\hline USDA/APHIS & Government & Agriculture & https://www.aphis.usda.gov/aphis/home/ \\
\hline $\begin{array}{l}\text { Department for Environment, Food and Rural } \\
\text { Affairs (UK) }\end{array}$ & Government & Agriculture & $\begin{array}{l}\text { https://www.gov.uk/government/organisations/ } \\
\text { department-for-environment-food-rural-affairs }\end{array}$ \\
\hline Transport Canada & Government & Aviation & http://www.tc.gc.ca/eng/menu.htm \\
\hline USFWS & Government & Energy/aviation/buildings & $\begin{array}{l}\text { https://www.fws.gov/birds/bird-enthusiasts/threa } \\
\text { ts-to-birds/collisions.php }\end{array}$ \\
\hline Bureau of Ocean Energy Management & Government & Energy & https://www.boem.gov/ \\
\hline $\begin{array}{l}\text { USDA National Wildlife Research Center-Staff } \\
\text { Publications }\end{array}$ & Government & Agriculture & http://digitalcommons.unl.edu/icwdm_usdanwrc/ \\
\hline Bird Strike Association of Canada & $\begin{array}{l}\text { Government/ } \\
\text { Industry Part- } \\
\text { nership }\end{array}$ & Aviation & http://www.canadianbirdstrike.ca/en \\
\hline British Trust for Ornithology & Non-profit & Bird conservation & https://www.bto.org/ \\
\hline Bird-life International & Non-profit & Bird conservation & http://www.birdlife.org/ \\
\hline FLAP & Non-profit & Bird/window collision & http://www.flap.org/who-we-are.php \\
\hline International Dark Skies Association & Non-profit & Conservation & http://darksky.org/ \\
\hline Internet Center for Wildlife Damage Management & Non-profit & Human-wildlife conflict & http://digitalcommons.unl.edu/icwdm/ \\
\hline IUCN SSC Human Wildlife Conflict Task Force & Non-profit & Human-wildlife conflict & http://www.hwctf.org/resources/document-library \\
\hline Proceedings of the Vertebrate Pest Conference & Academic & Human-wildlife conflict & $\begin{array}{l}\text { http://www.vpconference.org/Proceedings_of_- } \\
\text { the_Vertebrate_Pest_Conference/ }\end{array}$ \\
\hline
\end{tabular}

we will disable browser history and cookies when conducting searches. The search team will use "incognito mode" in Google Chrome and not access any electronic accounts during the search session.

\section{Language restrictions}

Searches will not be restricted by language, but only articles written in or translated to English will be included in the systematic map.

\section{Search records}

For each database or citations indexing service search, the date, search string, license used, and number of results will be recorded and reported in the final systematic map. When possible, the search results from each database will be exported and saved in a Zotero library. The search results from each database will be made available in an .RIS and BibLaTex format as a supplement to the Systematic Map. The Web of Science Core Collection search results will be imported into Excel as described above in the "Increasing specificity" section. The search results of all other exportable searches will be exported from Zotero to a Microsoft Excel spreadsheet and will be combined with the search results from the Web of Science Core Collection Search. All articles with the same title, authors, and journal name will be considered duplicates and one of the duplicates will be removed from the spreadsheet. The final spreadsheet of search results will be included as a supplement in the Systematic Map.

Some databases and most websites lack options to export search results. When possible, we will copy and paste the search results into a Microsoft Word document. We will record search dates and save relevant articles in a Zotero library. The Microsoft Word documents showing all search results and the Zotero library of relevant articles will also be published as a supplement to the Systematic Map.

\section{Assessing search comprehensiveness}

We developed the search strategy to ensure that all benchmark articles (Additional file 2) will be found in the search. Benchmark articles include articles known to the authors, articles found in relevant reviews, and additional articles found during scoping. After all searches are complete, we will confirm that the search strategy finds all benchmark articles. 
We will further assess the search comprehensiveness through bibliographic checking of a review pertaining to each secondary question. For each secondary question, if at least one review is identified in our search, we will randomly select one review for bibliographic checking. We will record the bibliographic details for the review, the number of references assessed as relevant in the review's reference list, and the number of relevant references missed by our searches. We will add additional components to the search strategy until all relevant references are found.

\section{Article screening and study eligibility criteria Screening process}

Articles will be screened for relevance in Microsoft Excel using the eligibility criteria outlined in Table 5 . The screening process will occur in two stages: title/abstract and full text. If at the title/abstract stage the relevance of the article cannot be determined, it will be included in the full text stage of screening. The full text of all articles not excluded at the title or abstract stages will be accessed using the licenses of the University of Alberta and Purdue University. Any full texts that are not available through these licenses will be obtained through Google Scholar or will be requested from the authors. The number of articles excluded at each stage will be listed in a PRISMA flow chart [48]. For each article excluded at the full text stage, the reasons for exclusion will be recorded in Excel and reported.
One article may contain multiple studies, and the same study may be reported in multiple articles. During title/ abstract and full text screening we will screen for eligibility at the article level. The systematic map will include a Microsoft Access database with two separate tables: Articles and Studies (see Data Coding Strategy). We will include any article that contains at least one eligible study in the Articles table. If an article containing an eligible study also contains ineligible studies, we will exclude the ineligible studies from the Studies table. Study eligibility will be determined during data coding, and reasons for exclusion of the ineligible studies will be recorded in the Articles table.

\section{Screening consistency checking}

Two members of the mapping team (CAA and $A B$ ) will screen and evaluate the same random subset of 200 articles at the title/abstract level and 20 articles at the full text stage. We will complete the consistency checking exercise at the title/abstract level before beginning screening and repeat the process after 7000 articles have been screened to maintain confidence in comparable judgement. Although greater consistency might be achieved if two researchers screened every article [49], screening by single authors is necessary to screen such a high number of expected search results $(>15,000)$. We will measure consistency of eligibility decisions between the researchers with the Kappa test. After consistency checking, the mapping team will discuss discrepancies and clarify the

Table 5 Eligibility criteria

\begin{tabular}{|c|c|c|}
\hline & Eligibility criteria & Exclusion criteria \\
\hline Population & Any bird species, including domesticated species & \\
\hline Intervention/exposure & $\begin{array}{l}\text { Anthropogenic light sources, including, but not limited to, point sources of light, } \\
\text { illuminated habitat, and skyglow }\end{array}$ & \\
\hline Comparator & $\begin{array}{l}\text { Same site under different lighting conditions } \\
\text { Nearby site with similar habitat type, anthropogenic activity, migration density } \\
\text { (jf applicable) and weather as the treatment site, at the same time of day } \\
\text { Documentation of aggregation, mass landing, mortality, or other bird behav- } \\
\text { iours near artificial light sources without comparator will be included in the } \\
\text { map and categorized as incidental reports }\end{array}$ & \\
\hline Outcome & $\begin{array}{l}\text { Bird density, including (but not limited to) radar observations, visual counts and } \\
\text { vocalization counts } \\
\text { Bird mortality } \\
\text { Resource consumption (such as crop damage or aquaculture predation) } \\
\text { Behavioural outcomes directly involving movement through space, including } \\
\text { (but not limited to) orientation, flight path, flight initiation, diving and foraging } \\
\text { Behaviours known to precede movement: overt reaction distance, alert } \\
\text { response (e.g. moving head laterally or vertical, crouching) } \\
\text { Temporal shifts in behaviours involving movement through space or habitat use } \\
\text { (e.g. migrating, foraging, reproduction) }\end{array}$ & $\begin{array}{l}\text { Responses that are not conventionally } \\
\text { associated with movement (e.g. hormo- } \\
\text { nal responses) } \\
\text { Behavioural responses that do not include } \\
\text { movement through space (e.g. preen- } \\
\text { ing) }\end{array}$ \\
\hline Study design & $\begin{array}{l}\mathrm{BA}, \mathrm{Cl}, \mathrm{BACl}, \mathrm{RCT} \text {, time-series, post hoc surveys, or description/anecdote (e.g. } \\
\text { documentation of mass mortality event) }\end{array}$ & \\
\hline
\end{tabular}

Articles containing eligible studies will be included in the map. Studies must meet one of the eligibility criteria in each category to be included in the map, and studies that meet any of the exclusion criteria will not be included in the map 
eligibility criteria. If the Kappa score is $<0.6, \mathrm{CAA}$ or $\mathrm{AB}$ will use the new eligibility criteria to repeat the consistency checking exercise on an additional random subset at both screening stages. Changes to the eligibility criteria will be reported in the map. Consistency checking for eligibility screening at the study level will be performed during consistency checking of the data coding strategy (see below).

\section{Study validity assessment}

We will not critically appraise overall study validity. The heterogeneity in study design, populations, interventions/exposures, and outcomes included in the scope of the map does not support consistent criteria for evaluating studies. However, study design will be categorized as BACI, BA, CI, CI-rotating, behavioural assay, habitat/resource selection, or incidental report. Description of the study methods and replicates will be recorded to aid in identifying subtopics that may support systematic review.

\section{Data coding strategy}

The systematic map will include a Microsoft Access database with metadata about each article and study, as outlined in Table 6. We will extract a broad range of metadata, including treatments, effect modifiers (e.g. weather, ambient light, habitat, temporal variables etc.), outcomes measured, and outcome measurement methods. Table 6 lists the metadata that will be included in the Articles table in the database, and Table 7 lists metadata included in the Studies table. Changes to these categories and variables will be reported in the systematic map. The database will have search and filter functions to identify studies with any combination of category values.

During full text data extraction, any metadata that cannot be obtained will be coded as "UA" (unattainable). Any metadata category that is not applicable to a study will be coded as "NA" (not applicable). Studies for which full text is not available after contacting the author will be included in the database, with the category Full Text Available coded as "No" and all metadata which is not available in the abstract will be coded as "UA."

\section{Data coding consistency checking}

To ensure that data are being extracted in a consistent and repeatable manner, data for at least ten studies will be extracted by two researchers (AB and $\mathrm{CA}$ ) and compared to check consistency. If any inconsistencies occur, the mapping team will discuss the discrepancies and provide additional specifications in the Description columns of the Articles and Studies tables if necessary. These additional specifications will be recorded and reported in the systematic map.

\section{Study mapping and presentation}

We will publish the database generated by the systematic map as an additional file to the published manuscript in the form of a Microsoft Access database. Both the Articles and Studies tables will also be available as Microsoft Excel files. We will present the data so that users that have basic familiarity with Microsoft Excel can use simple filters to explore the product of the systematic map.

The narrative report, supported by heat maps, will describe the volume and key characteristics of the

Table 6 Articles table data extraction categories and values

\begin{tabular}{|c|c|c|}
\hline Data extraction category & Description & $\begin{array}{l}\text { Drop-down options (additional options may be generated } \\
\text { during data extraction) }\end{array}$ \\
\hline ID & Unique number assigned to each paper & \\
\hline Citation & CSE style in text citation & \\
\hline \multicolumn{3}{|l|}{ Title } \\
\hline \multicolumn{3}{|l|}{ Year of publication } \\
\hline Publication venue & Journal name, conference, etc. & \\
\hline Publication type & & $\begin{array}{l}\text { Academic journal; book chapter; conference proceedings; gov- } \\
\text { ernment agency publication; industry publication; non-profit } \\
\text { publication; magazine article; thesis }\end{array}$ \\
\hline Peer-reviewed & & Yes/no \\
\hline Full text available to authors & & Yes/no \\
\hline \multicolumn{3}{|l|}{ Language } \\
\hline Contains excluded studies & & Yes/no \\
\hline PICO/PECO reasons for exclusion & & Population; intervention/exposure; outcome \\
\hline Reasons for exclusion notes & $\begin{array}{l}\text { Briefly describe the reasons any studies within } \\
\text { the article were excluded from the Studies } \\
\text { table }\end{array}$ & \\
\hline
\end{tabular}




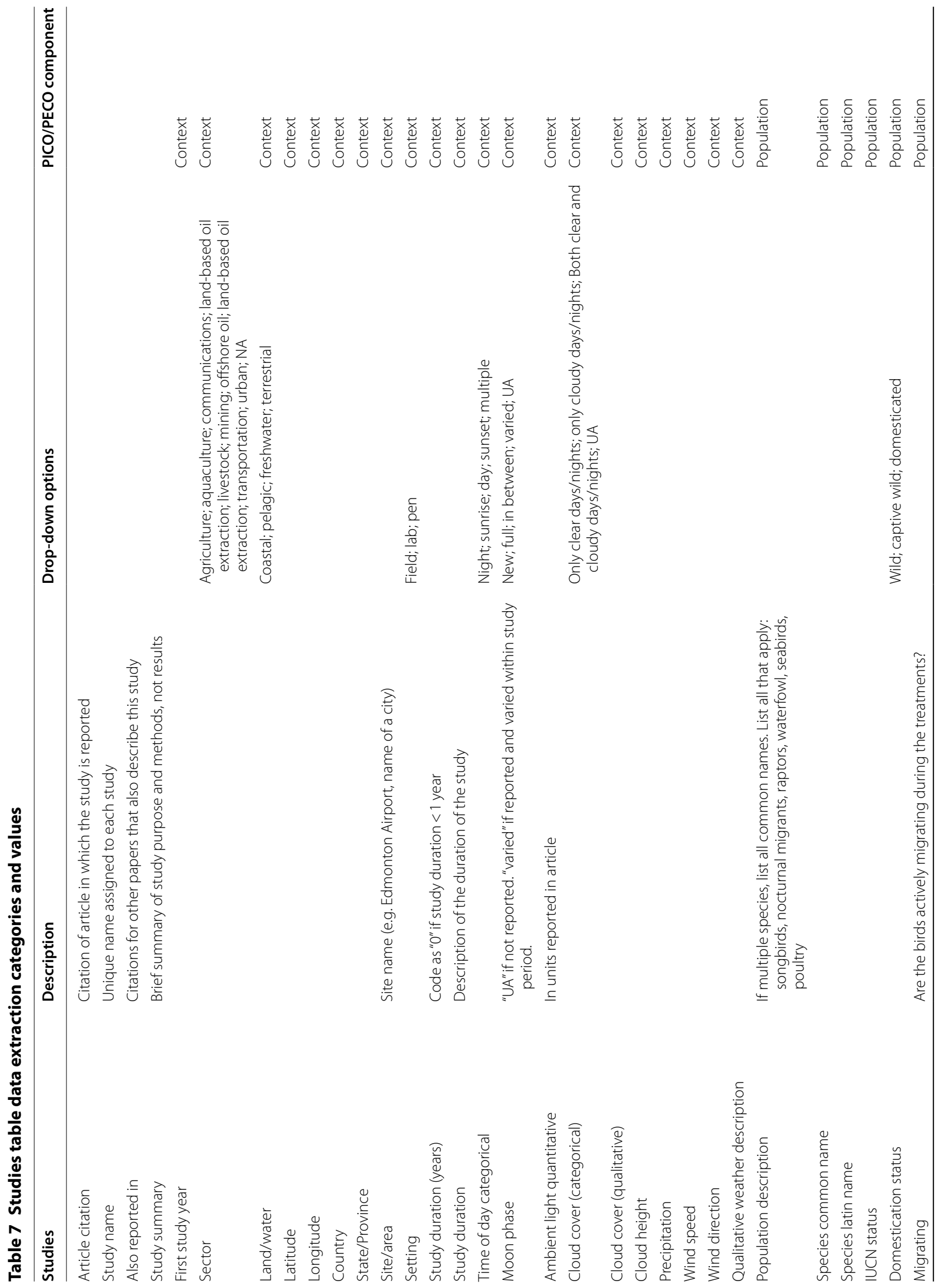



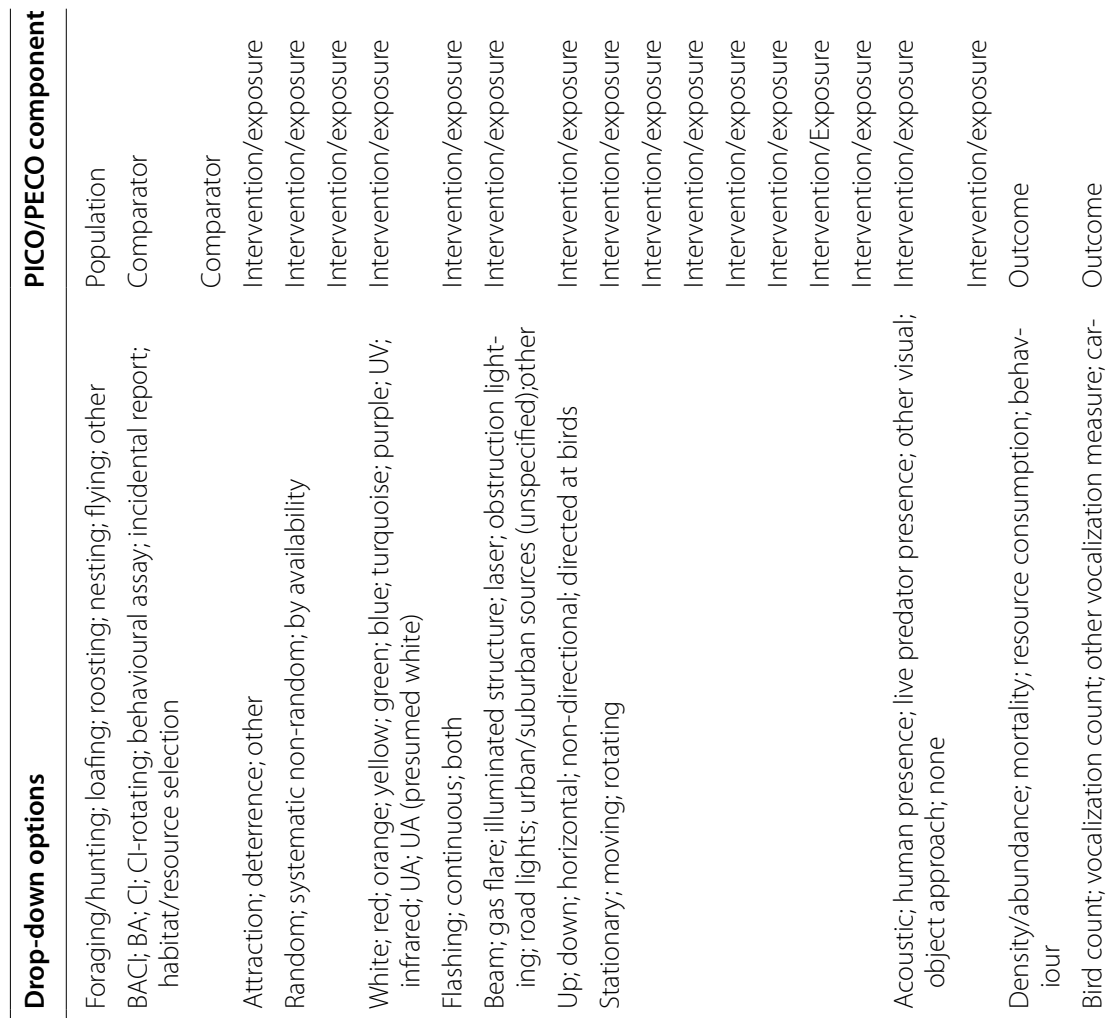

¿ั
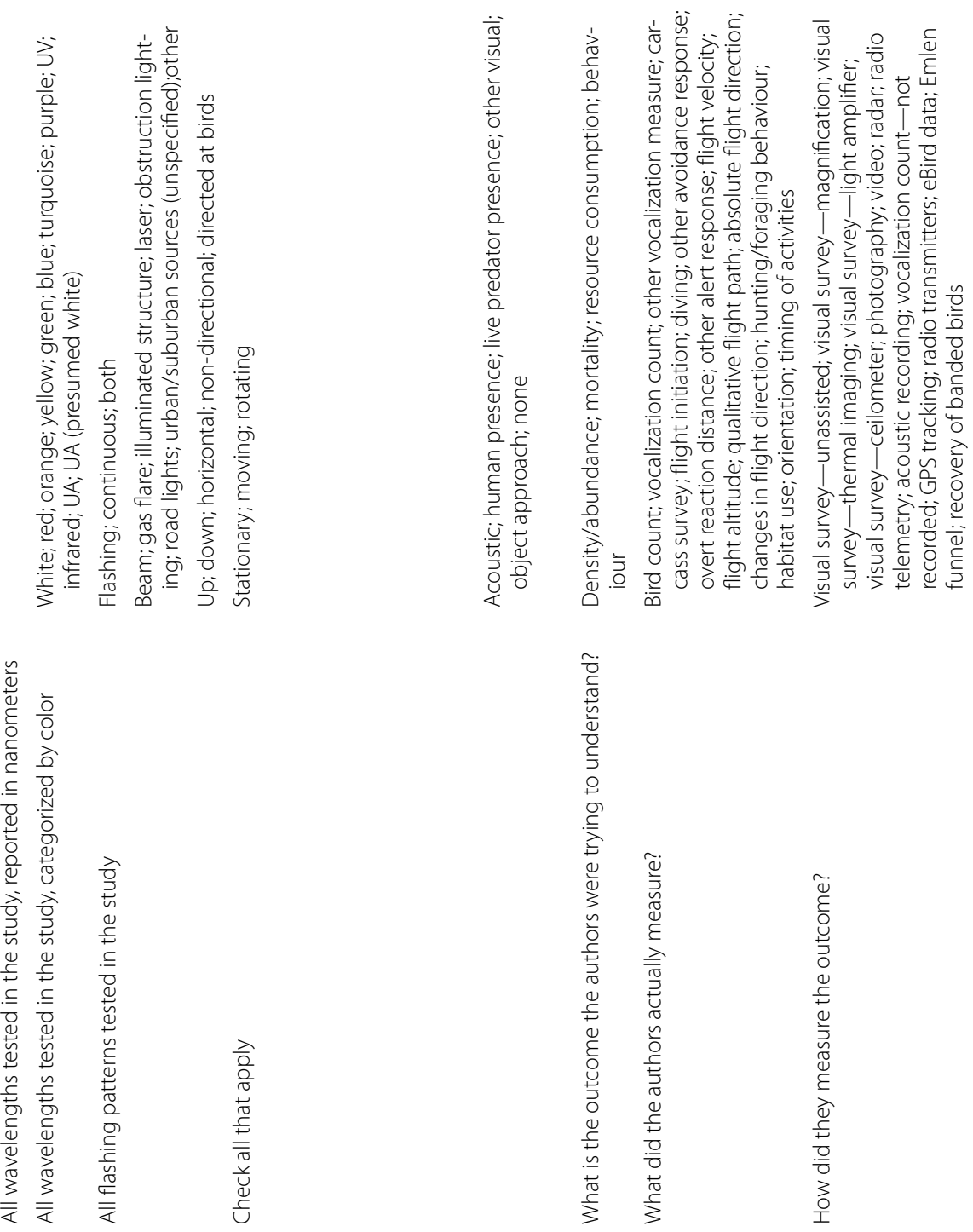

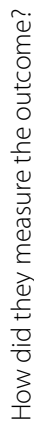
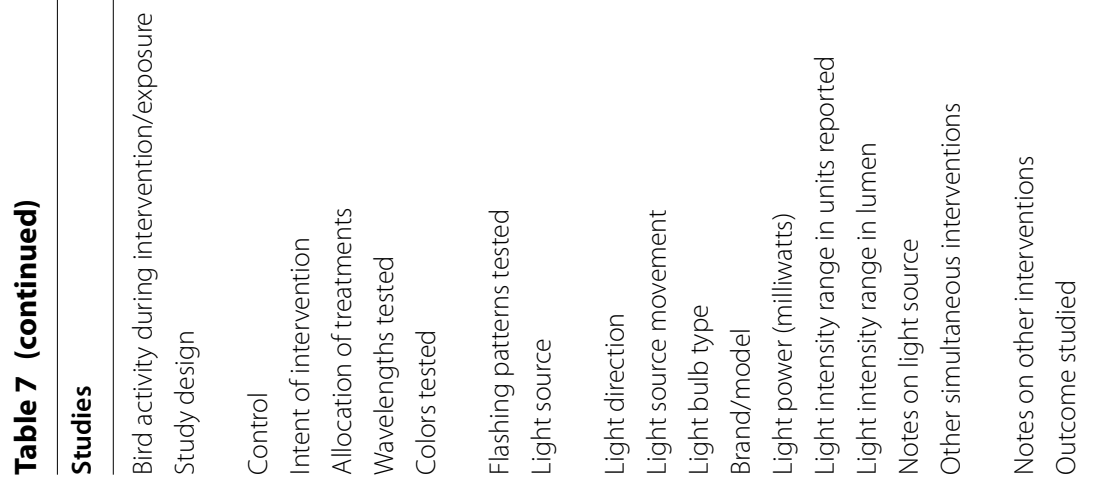

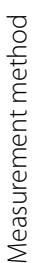




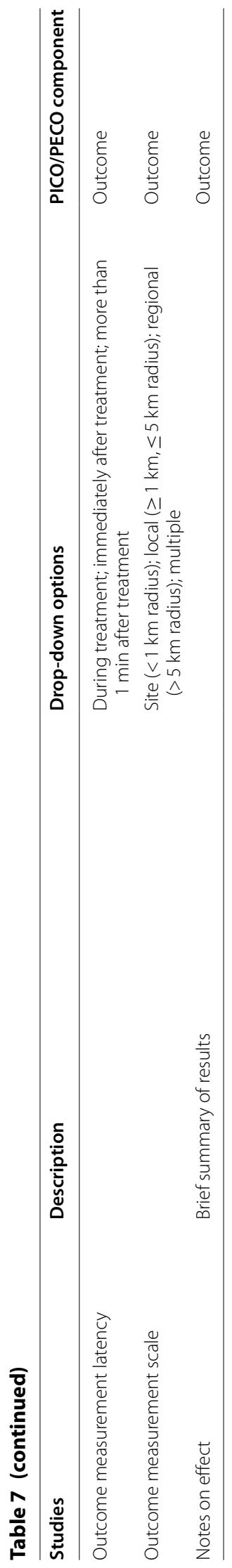


evidence base. The report will identify subtopics that require further primary research (knowledge gaps) and subtopics that may have been sufficiently studied to allow a systematic review (evidence clusters). We will describe the number and study design of studies pertaining to the following three subtopics:

- How does artificial light associated with illuminated anthropogenic structures affect bird movement or aggregation?

- Are light-based deterrents effective at deterring birds?

- How does artificial light affect bird density or habitat use?

Additional subtopics may be identified through the course of producing the systematic map. We will describe the following sources of heterogeneity among studies within each subtopic: light characteristics (e.g. wavelength, intensity, direction, and flashing pattern), environmental variables (e.g. weather variables, temporal variables, moon phase, land/freshwater/ocean), and population characteristics (e.g. species, bird activity during intervention/exposure, domestication status, migratory status).

By identifying knowledge gaps evidence clusters, describing the comparability of outcomes measured within each subtopic, and identifying stakeholder interest, we will provide critical information that we and other authors may use to identify systematic review topics and guide primary research. The relative number of studies, excluding incidental reports, for these subtopics and sources of heterogeneity will be visualized as heat maps and narratively described. Subtopics investigated by relatively few studies will be listed as knowledge gaps, while those represented in the greatest number of studies will be listed as evidence clusters. We anticipate that a lack of comparable outcomes across studies will be a barrier to systematic review. Within each of the subtopics, we will characterize the outcomes measured (e.g. bird density, mortality, qualitative flight path, alert response) to provide information on whether studies can be systematically compared and encourage greater standardization of measurement in future. This narrative report, together with feedback from surveyed stakeholders, will help inform the choice of subsequent subtopics for systematic review and/or meta-analysis.

\section{Additional files}

Additional file 1. Stakeholder survey.

Additional file 2. Benchmark articles.

Additional file 3. Expanded population search string.
Authors' contributions

CAA, AB, CCS, and EFJ collectively identified the topic for the systematic map and developed the search and data extraction strategies. All authors contributed to the development of the stakeholder engagement strategy. CAA wrote the protocol manuscript with significant contributions from $A B, C C S$, and EFJ. EB also edited the protocol and advised in the development of the database. All authors agreed on the final version of the protocol. All authors read and approved the final manuscript.

\section{Author details \\ ${ }^{1}$ Department of Biological Science, University of Alberta, CW 405, Biological Sciences Building, Edmonton, AB T6G 2E9, Canada. ${ }^{2}$ Department of Biological Sciences, Purdue University, 915 W. State Street, West Lafayette, IN 47907, USA.}

\section{Acknowledgements}

We extend our gratitude to the many people and institutions that contributed to training $C A A$ and $A B$ in systematic evidence synthesis workshop tailored for behavioural ecologists at the Jacob Blaustein Institutes for Desert Research, Ben- Gurion University of the Negev, Israel. The workshop was taught by Biljana Macura (MISTRA-EviEM and Stockholm Environment Institute, Sweden) and organized by Oded Berger-Tal, Alison Greggor, and Daniel Blumstein. The workshop was made possible through collaboration between Ben-Gurion University and Monash University, Australia, as well as generous support from the Jacob Blaustein Center for Scientific Cooperation, The Swiss Institute for Dryland Environmental \& Energy Research, Mitrani Department of Desert Ecology, and Ben-Gurion University of the Negev.

\section{Competing interests}

The authors declare that they have no competing interests. No member of the mapping team will be involved screening or extracting data from a study on which he or she is an author.

\section{Availability of data and materials \\ Not applicable.}

\section{Consent for publication}

Not applicable.

\section{Ethics approval and consent to participate}

The authors consulted with the University of Alberta Research Ethics Office and determined that no ethics approval is required for this study.

\section{Funding}

Graduate student stipends, training in systematic review methodology, and publication costs of the systematic map protocol are funded by NSERC Discovery Grants and Research Fellowships from the Faculty of Science, University of Alberta to CCSC and EB and through the National Fish and Wildlife Foundation and Packard Foundation to EFJ.

\section{About this supplement}

This article has been published as part of Environmental Evidence Volume 8 Supplement 1, 2019: Using animal behavior in conservation management. The full contents of the supplement are available online at https://environmen talevidencejournal.biomedcentral.com/articles/supplements/volume-8-suppl ement-1.

\section{Publisher's Note}

Springer Nature remains neutral with regard to jurisdictional claims in published maps and institutional affiliations.

Published: 13 June 2019

\section{References}

1. Kyba CCM, Kuester T, De Miguel AS, Baugh K, Jechow A, Hölker F, et al. Artificially lit surface of Earth at night increasing in radiance and extent. Sci Adv. 2017;3:1-9. 
2. Gauthreux SA, Belser CG. Effects of artificial night lighting on migrating birds. In: Longcore T, Rich C, editors. Ecological consequences of artificial night lighting. Washington, DC: Island Press; 2006. p. 67-94.

3. Bishop JD, McKay HV, Parrott D, Allan J. Review of international research literature regarding the effectiveness of auditory bird scaring techniques and potential alternatives. United Kingdom; 2003.

4. Day RH, Rose JR, Prichard AK, Streever B. Effects of gas flaring on the behavior of night-migrating birds at an artificial oil-production Island, Arctic Alaska. Arct Inst North Am. 2015;68:367-79.

5. Glahn JF, Ellis G, Fioranelli P, Dorr BS. Evaluation of moderate and lowpowered lasers for dispersing double-crested cormorants form their night roosts. In: Proceedings of the 9th wildlife damage management conference. 2000. p. 34-45.

6. Read JL. A strategy for minimizing waterfowl deaths on toxic waterbodies. J Appl Ecol. 1999;36:345-50.

7. McLaren JD, Buler JJ, Schreckengost T, Smolinsky JA, Boone M, Emiel van Loon E, et al. Artificial light at night confounds broad-scale habitat use by migrating birds. Ecol Lett. 2018;21:356-64.

8. Blackwell BF, Fernandez-Juricic E. Behavior and physiology in the development and application of visual deterrents at airports. In: DeVault T, Blackwell B, Belant J, editors. Preventing animal-aircraft collisions through science-based management. Baltimore: The Johns Hopkins University Press, in association with The Wildlife Society; 2013. p. 11-22.

9. Cabrera-Cruz SA, Smolinsky JA, Buler JJ. Light pollution is greatest within migration passage areas for nocturnally-migrating birds around the world. Sci Rep. 2018;8:3261. https://doi.org/10.1038/s41598-01821577-6.

10. Atzeni M, Fielder D, Thomson B. Deterrence of wild waterfowl from poultry production areas: a critical review of current techniques and literature. 2016.

11. Marsh RE, Erickson WA, Salmon TP. Bird hazing and frightening methods and techniques. Davis, California; 1991. http://digitalcommons.unl. edu/icwdmother/51\%. Accessed 14 Dec 2017.

12. Harris RE, Davis RA. Evaluation of the efficacy of products and techniques for airport bird control. King City, Ontario, Canada; 1998. http://www.tc.gc.ca/publications/bil/TP13029/PDF/HR/TP13029B.pdf. Accessed 14 Dec 2017.

13. Ronconi RA, Allard KA, Taylor PD. Bird interactions with offshore oil and gas platforms: review of impacts and monitoring techniques. J Environ Manag. 2015;147:34-45. https://doi.org/10.1016/j.jenvm an.2014.07.031.

14. Kerlinger P, Gehring JL, Erickson WP, Curry R, Jain A, Guarnaccia J. Night migrant fatalities and obstruction lighting at wind turbines in north america. Wilson J Ornithol. 2010;122:744-54. https://doi. org/10.1676/06-075.1.

15. Longcore T, Rich C, Gauthreaux SA. Height, guy wires, and steadyburning lights increase hazard of communication towers to nocturnal migrants: a review and meta-analysis. Auk. 2008;125:485-92. https:// doi.org/10.1525/auk.2008.06253.

16. Calvert AM, Bishop CA, Elliot RD, Krebs EA, Kydd TM, Machtans CS, et al. A synthesis of human-related avian mortality in canada. Avian Conserv Ecol. 2013:8:11.

17. Ogden LJE. Summary report on the bird friendly building program: effect of light reduction on collision of migratory birds. 2002.

18. Rodríguez A, Burgan G, Dann P, Jessop R, Negro JJ, Chiaradia A. Fatal attraction of short-tailed shearwaters to artificial lights. PLOS ONE. 2014;9:e110114.

19. Van Doren BM, Horton KG, Dokter AM, Klinck H, Elbin SB, Farnsworth A. High-intensity urban light installation dramatically alters nocturnal bird migration. Proc Natl Acad Sci. 2017;1 14:11175-80. https://doi. org/10.1073/pnas.1708574114

20. Day RH, Prichard AK, Rose JR, Streever B, Swem T. Effects of a hazinglight system on migration and collision avoidance of eiders at an artificial oil-production Island, Arctic Alaska. Arctic. 2017;70:13-24.

21. Jones HP. The effect on birds of a North Sea gas flare. Br Birds. 1980;73:547-55.

22. Evans WR, Akashi Y, Altman NS, Manville AM II. Response of nightmigrating songbirds in cloud to colored and flashing light. North Am Birds. 2007;60:476-88.
23. Poot $H$, Ens BJ, de Vries H, Donners MA, Wernand MR, Marqueni JM. Green light for nocturnally migrating birds. Ecol Soc. 2008;13:47. https ://www.ecologyandsociety.org/vol13/iss2/art47/.

24. Larkin RP, Frase BA. Circular paths of birds flying near a broadcasting tower in cloud. J Comp Psychol. 1988;102:90-3. https://doi. org/10.1037/0735-7036.102.1.90.

25. Blackwell BF, Bernhardt GE, Dolbeer RA. Lasers as nonlethal avian repellents. J Wildl Manag. 2002;66:250-8.

26. Goller B, Blackwell BF, DeVault TL, Baumhardt PE, Fernández-Juricic E. Assessing bird avoidance of high-contrast lights using a choice test approach: implications for reducing human-induced avian mortality. PeerJ. 2018;6:e5404. https://doi.org/10.7717/peerj.5404.

27. Andelt WF, Woolley TP, Hopper SN. Effectiveness of barriers, pyrotechnics, flashing lights, and Scarey Man for deterring heron predation on fish. Wildl Soc Bull. 1997;25:686-94.

28. Avery ML, Humphrey JS, Tillman E a, Milleson MP. Responses of Black Vultures to roost dispersal in Radford, Virginia. In: Proceedings of the Vertebrate Pest Conference 2006; April 2004:239-43.

29. Verheijen FJ. The mechanisms of the trapping effect of artificial light sources upon animals. Arch Néerlandaises Zool. 1960;13:1-107. https:// doi.org/10.1163/036551660X00017.

30. Harris MD, Lincoln AE, Amoroso PJ, Stuck B, Sliney D. Laser eye injuries in military occupations. Aviat Sp Environ Med. 2003;74:947-52.

31. Barkana Y, Belkin M. Laser eye injuries. Surv Ophthalmol. 2000;44:459-78.

32. Gunduz K, Arden GB. Changes in colour contrast sensitivity associated with operating argon lasers. Br J Ophthalmol. 1989;73:241-6.

33. Wiltschko R, Stapput K, Thalau P, Wiltschko W. Directional orientation of birds by the magnetic field under different light conditions. J R Soc Interface. 2010;7(Suppl_2):163-77. https://doi.org/10.1098/ rsif.2009.0367.focus.

34. Rappl R, Wiltschko R, Weindler P. Orientation behavior of Garden Warblers (Sylvia borin) under monochromatic light of various wavelengths. Auk. 2000;117:256-60.

35. Fernandez-Juricic $E$. The role of animal sensory perception in behaviorbased management. In: Saltz D, Berger-Tal O, editors. Conservation behavior: applying behavioural ecology to wildlife conservation and management. Cambridge: Cambridge University Press; 2016. p. $149-75$.

36. Fernández-Juricic E. Sensory basis of vigilance behavior in birds: synthesis and future prospects. Behav Processes. 2012;89:143-52.

37. Cuthill IC. Color perception. In: Hill GE, McGraw KJ, editors. Bird coloration. Cambridge: Harvard University Press; 2006. p. 3-40.

38. Tanaka KD. A colour to birds and to humans: why is it so different? J Ornithol. 2015;156:433-40. https://doi.org/10.1007/s10336-015-1234-1.

39. Jones MP, Pierce KE, Ward D. Avian vision: a review of form and function with special consideration to birds of prey. J Exot Pet Med. 2007;16:69-87.

40. Conover M. Fear-provoking stimuli. In: Conover M, editor. Resolving human-wildlife conflicts: the science of wildlife damage management. Boca Raton: CRC Press; 2001. p. 229-47.

41. Gehring J, Kerlinger P, Manville AM. Communication towers, lights, and birds: successful methods of reducing the frequency of avian collisions. Ecol Appl. 2009;19:505-14.

42. Audubon Light Out. https://www.audubon.org/conservation/project/ lights-out. Accessed 7 Dec 2018.

43. Orr TL, Herz SM, Oakley DL. Evaluation of lighting schemes for offshore wind facilities and impacts to local environments. Rep Prep under BOEM Contract M12-PD-00007. 2013;431. https://www.fbo.gov/index

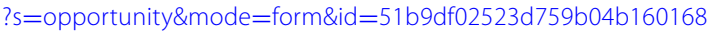
$567 \mathrm{~b} 62 \mathrm{f} \& \mathrm{tab}=\mathrm{core} \& \_\mathrm{cview}=0$.

44. Holevinski RA, Curtis PD, Malecki RA. Human-Wildlife interactions wildlife damage management, internet center for Hazing of Canada geese is unlikely to reduce nuisance populations in urban and suburban communities Hazing of Canada geese is unlikely to reduce nuisance populations in urban and Human-Wildlife Conflicts. 2007;1:257-64. http://digitalcommons.unl.edu/hwi\%0A http://digitalcommons.unl. edu/hwi/97.

45. Guidelines for Systematic Review and Evidence Synthesis in Environmental Management. Environ Evid. 2013;Version 4. 
46. Haddaway NR, Macura B, Whaley P, Pullin AS. ROSES reporting standards for systematic evidence syntheses: pro forma, flow-diagram and descriptive summary of the plan and conduct of environmental systematic reviews and systematic maps. Environ Evid. 2018;7:4-11. https://doi.org/10.1186/s13750-018-0121-7.

47. Gill F, Donsker D. IOC World Bird List v4.2. International ornithologists union committee on nomenclature. 2014. https://doi.org/10.14344/ioc ml.4.2.
48. Moher D, Liberati A, Tetzlaff J, Altman DG, Altman D, Antes G, et al Preferred reporting items for systematic reviews and meta-analyses: the PRISMA statement. PLoS Med. 2009;6:e1000097.

49. Frampton GK, Livoreil B, Petrokofsky G. Eligibility screening in evidence synthesis of environmental management topics. Environ Evid. 2017;6:1-13.
Ready to submit your research? Choose BMC and benefit from:

- fast, convenient online submission

- thorough peer review by experienced researchers in your field

- rapid publication on acceptance

- support for research data, including large and complex data types

- gold Open Access which fosters wider collaboration and increased citations

- maximum visibility for your research: over 100M website views per year

At BMC, research is always in progress.

Learn more biomedcentral.com/submissions 